\title{
Corrigendum: QUICKstep and GS-TAP: new moves for protein-interaction analysis
}

Pascal Braun, Michael E Cusick \& Marc Vidal

Nat. Methods 3, 975-976 (2006).

In the version of this News \& Views article originally published, the RNAi control cell in Figure 1a was mislabeled as " $\left[{ }^{14} \mathrm{C}\right]$ arginine-labeled." The correct label should be "RNAi control: $\left[{ }^{13} \mathrm{C}\right]$ arginine-labeled." 